\title{
DESIGN AND ANALYSIS OF EXPERIMENTS
}


This page intentionally left blank 


\section{DESIGN AND ANALYSIS OF EXPERIMENTS}

\section{Arjun K Gupta}

Bowling Green State University, USA

D G Kabe 
Published by

World Scientific Publishing Co. Pte. Ltd.

5 Toh Tuck Link, Singapore 596224

USA office: 27 Warren Street, Suite 401-402, Hackensack, NJ 07601

UK office: 57 Shelton Street, Covent Garden, London WC2H 9HE

\section{British Library Cataloguing-in-Publication Data}

A catalogue record for this book is available from the British Library.

\section{DESIGN AND ANALYSIS OF EXPERIMENTS}

Copyright (C) 2013 by World Scientific Publishing Co. Pte. Ltd.

All rights reserved. This book, or parts thereof, may not be reproduced in any form or by any means, electronic or mechanical, including photocopying, recording or any information storage and retrieval system now known or to be invented, without written permission from the Publisher.

For photocopying of material in this volume, please pay a copying fee through the Copyright Clearance Center, Inc., 222 Rosewood Drive, Danvers, MA 01923, USA. In this case permission to photocopy is not required from the publisher.

ISBN 978-981-4522-53-3

Printed in Singapore 


\title{
Dedication
}

\author{
For Samik \\ The Indo-Irish Significant Boy \\ AKG
}


This page intentionally left blank 


\section{Preface}

Design of experiments holds a central place in statistics. The aim of this book is to present in a readily accessible form certain theoretical results of this vast field. This is intended as a text book for a one-semester or two-quarter course for undergraduate seniors or first-year graduate students or as a resource of supplementary reading and references. Basic knowledge of algebra, calculus and statistical theory is required to master the techniques presented in this book. We believe that students and most teachers of design of experiments will find the book both interesting and useful.

The book contains 9 chapters. Chapter 1 provides the basic statistical tools that are used in the remaining chapters. It can be read first or can be used as reference for readers who can begin with Chapter 2. The main body of the material is presented in Chapters 2-7. Chapter 8 is different in the sense that it is pure mathematics and provides the elements of modern algebra which are used in Chapter 9. The last chapter discusses the construction of designs. The topics in a book as this always come from many sources. We are grateful to many teachers, students and authors who directly or indirectly have contributed to this book. Our special thanks go to our graduate students, $\mathrm{Hu}$ and Ngoc for their help in proofreading the manuscript and for suggesting many improvements.

The authors would like to acknowledge the support of the Bowling Green State University and the St. Mary's University. The sad demise of Professor D. G. Kabe during the preparation of this volume has delayed the completion of the book. We are 
thankful to Ms. Lai Fun Kwong and the staff of World Scientific for their help and cooperation in bringing this book to publication. Finally, we would like to thank Lei Kou and Nathan Baxter whose skills at word processing made the preparation of this book far less painful than it might have been. However, any errors or omissions remain entirely the responsibility of the authors.

Arjun K. Gupta

D. G. Kabe 


\section{Contents}

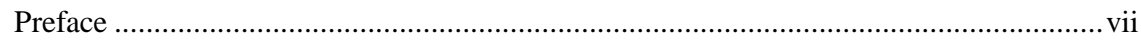

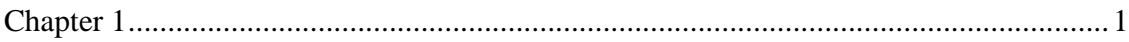

Introduction and Preliminary Results..................................................................... 1

1.1 Introduction: Elementary Statistical Notions ……........................................... 1

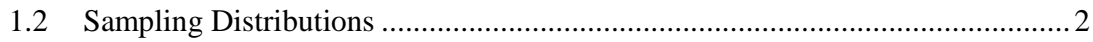

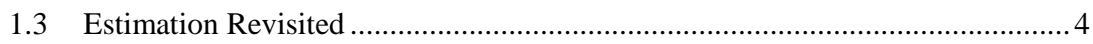

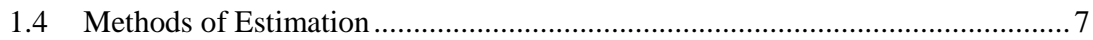

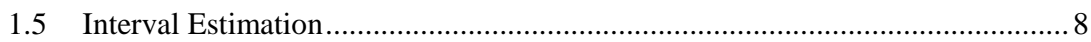

1.6 Linear Functions of Normally Distributed Variables ......................................... 9

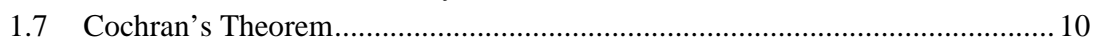

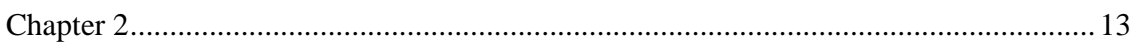

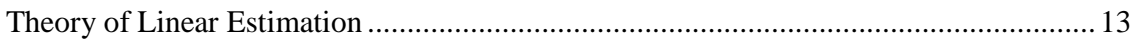

2.1 Basic Assumptions and Definition of Least Squares.......................................... 13

2.2 Linear Functions with Zero Expectation ......................................................... 21

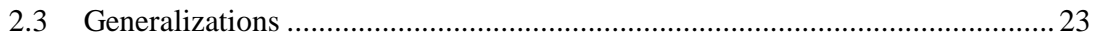

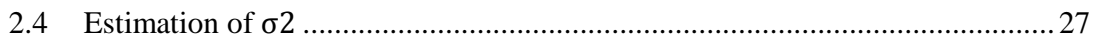

2.5 Observational Equations with Linear Restrictions On The Parameters..............31

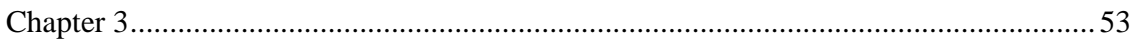

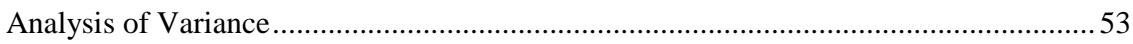

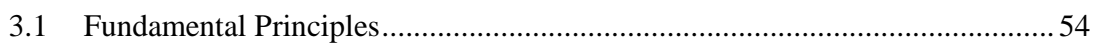

3.2 Partitioning of Sums of Squares and Degrees of Freedom...................................60

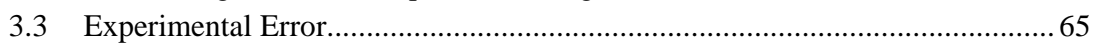

3.4 Assumptions Underlying the Analysis of Variance ............................................65

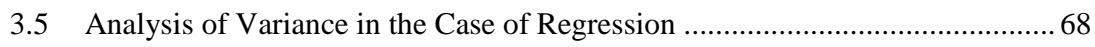

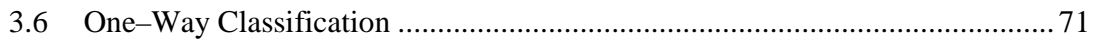

3.7 Two-Way Classification with a Single Observation per Cell ............................. 77

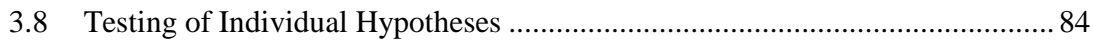

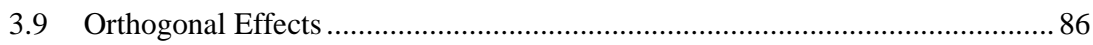


3.10 Two-Way Classification with Multiple but Equal Number of Observations in Cells.

3.11 Two-Way Classification with Multiple and Unequal Number of Observations in Cells ....................................................................................... 93

3.12 Matrix Methods (when there is no interaction) ............................................ 99

3.13 Case of Proportional Frequencies ................................................................. 104

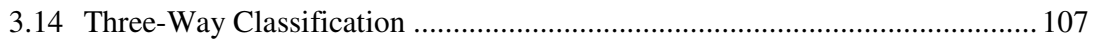

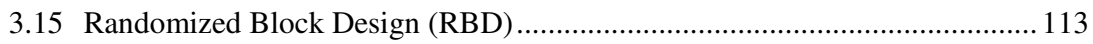

3.16 Judging the Relative Merits of Treatment ....................................................... 118

3.17 Confidence Limits for Treatment Differences ............................................. 118

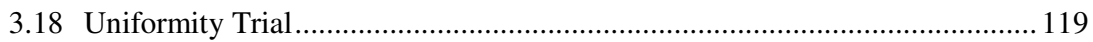

3.19 Latin Square Design.................................................................................. 120

3.20 Judging the Relative Merits of Treatments .................................................. 124

3.21 Graeco Latin Square ............................................................................... 125

3.22 Hyper - Graeco Latin Square .................................................................... 128

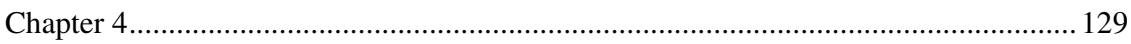

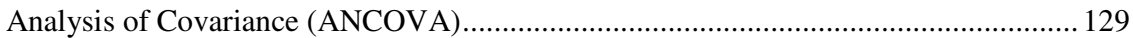

4.1 One-Way Classification with a Single Concomitant Variable ........................ 129

4.2 Two-Way Classification with One Observation per Cell and a Single

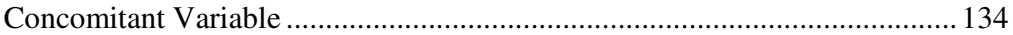

4.3 Some Examples of ANCOVA ….................................................................. 138

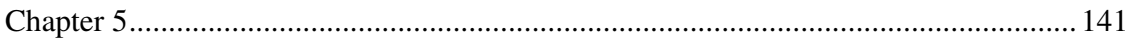

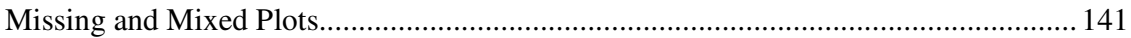

5.1 Two-Way Classification with One Cell Missing............................................ 141

5.2 Two-Way Classification with Two Missing Plots........................................... 144

5.3 K-Missing Plots: Yates' Method................................................................... 145

5.4 Bartlett's Method: Use of Concomitant Variables ........................................... 146

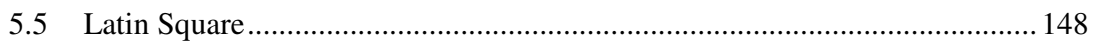

5.6 Randomized Block.............................................................................. 151

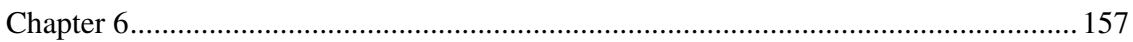

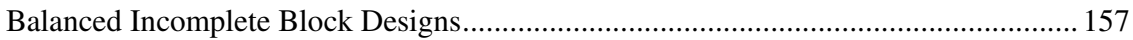

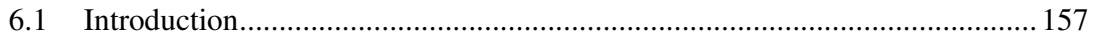

6.2 Symmetrical Balanced Incomplete Blocks..................................................... 159

6.3 Resolvable Balanced Incomplete Blocks ....................................................... 161

6.4 Affine Resolvable Balanced Incomplete Blocks .............................................. 164

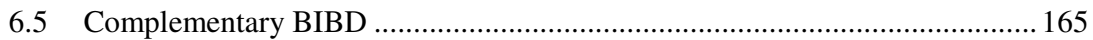

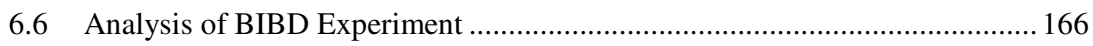




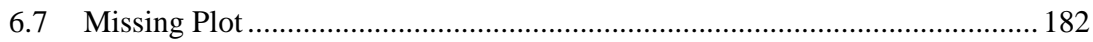

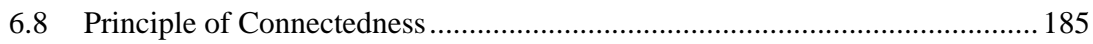

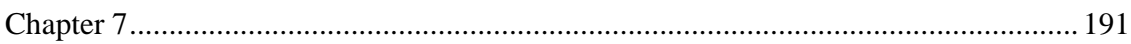

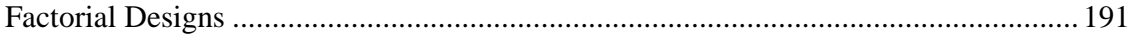

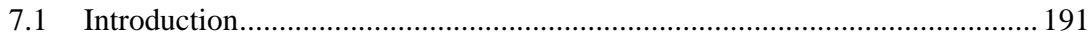

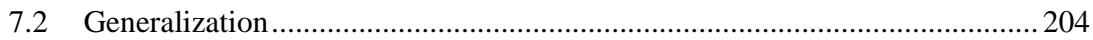

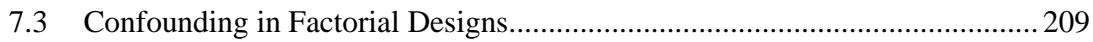

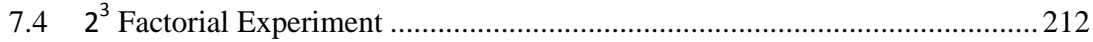

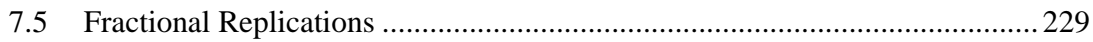

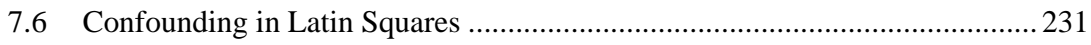

7.7 Partial Confounding ................................................................................. 233

Chapter 8

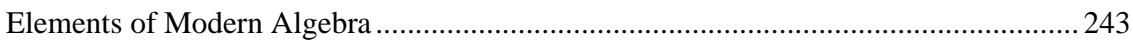

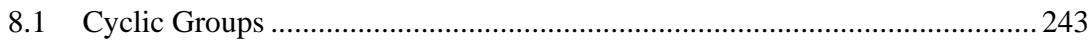

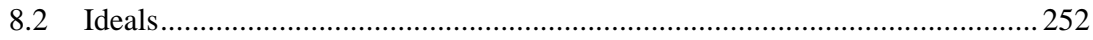

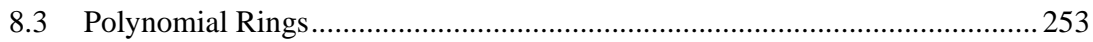

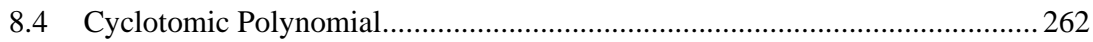

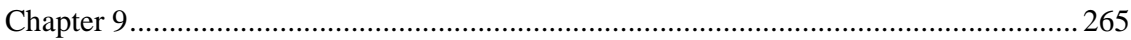

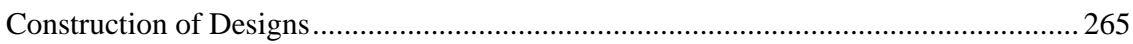

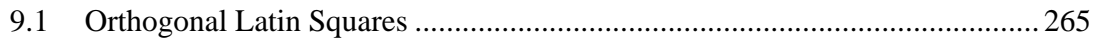

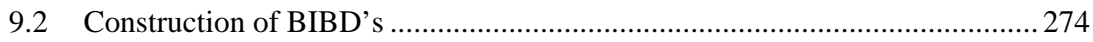

9.3 Geometric Method (Finite Projective Geometry).......................................... 280

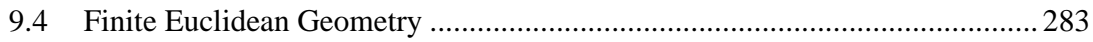

Index 